\title{
HT nach Mammakarzinom bleibt Einzelfallentscheidung
}

Fortschritte in der Therapie des Mammakarzinoms (Mamma-Ca) haben das Überleben der betroffenen Patientinnen deutlich verbessert. Damit einher gehen aber neue Fragestellungen. So leiden „knapp $75 \%$ aller postmenopausalen MammaCa-Patientinnen unter klimakterischen Beschwerden, die Hälfte davon schwerwiegend“, erklärte Prof. Dr. Cosima Brucker, Nürnberg. Der Frauenarzt steht damit vor einem schier unlösbaren Problem. Denn laut S3-Leitlinie ist eine Hormontherapie (HT) nach behandeltem Mamma-Ca wegen des erhöhten Rezidivrisikos kontraindiziert.

Brucker nahm daher die Datenlage genauer unter die Lupe. Danach zeigten ältere Studien kein erhöhtes Risiko, bei jedoch kleinen Fallzahlen. Mehr Aufschluss erwartete man sich von den beiden unabhängigen prospektiven randomisierten Untersuchungen, der HABITS-Studie mit $434 \mathrm{~Pa}$ tientinnen und der Stockholm-Studie $(\mathrm{n}=$ 378). HABITS (Hormonal Replacement therapy after breast cancer - is it safe?) wurde bereits nach 2,1 Jahren wegen eines signifikant erhöhten Rezidivrisiko (um das 3,3-Fache) beendet. 26 von 219 Frauen unter HT und 8 von 215 Frauen in der Kontrollgruppe hatten ein Rezidiv entwickelt. Anders erste Ergebnisse aus der StockholmStudie, die nach 4,1 Jahren keine Zunahme des Rezidivrisiko (HR 0,82) feststellte; wegen der HABITS-Resultate wurde sie jedoch vorzeitig beendet. Eine Interimsanalyse beider Studien ergab ein signifikant erhöhtes Mamma-Ca-Risiko unter HT mit einem mittleren Anstieg um das 1,8-Fache. Auch für Tibolon kann aufgrund der LIBERATE-Studie keine Entwarnung gegeben werden. Bei unselektierten MammaCa-Patientinnen war das Risiko 1,4-fach erhöht, bei Frauen mit rezeptorpositivem Mamma-Ca um das 1,56-Fache. Unter einem Aromataseinhibitor war die Gefahr höher als unter Tamoxifen (2,42 vs. 1,25). Bei rezeptornegativem Mamma-Ca stieg das Risiko dagegen nicht. Bruckers Fazit: „Die HT nach Mammakarzinom bleibt eine individuelle Einzelfallentscheidung in Ausnahmesituationen.“

Dr. Beate Fessler

2. Hauptthema "Kontrazeptiva/Hormone", 7. März 2012

\section{Brauchen wir Gensignaturtests beim frühen Mammakarzinom?}

Diese Frage, die Prof. Dr. Marion Kiechle, München, selbst in den Raum stellte, beantworte sie bereits zu Beginn ihres Vortrags mit „Ja! Wir brauchen sie zur Therapieentscheidung, ob eine Patientin von einer adjuvanten Chemotherapie profitiert." Bislang stützt sich eine Therapieentscheidung auf die klassischen Prognosefaktoren (z.B. Tumorgröße, Grading, Lymphknotenstatus). Gemäß dieser Konstellationen werden die Patientinnen in drei Risikokategorien - niedrig, mittel, hoch - (nach St. Gallen 2011 und S3Leitlinie) eingeteilt, in den nächsten zehn Jahren Fernmetastasen zu entwickeln, und daraus Behandlungsempfehlungen abgeleitet.

„In der großen Gruppe der Patientinnen mit intermediärem Risiko, insbesondere mit HER2-negativem Mammakarzinom, helfen uns die prognostischen Faktoren, die wir aktuell zur Verfügung haben, nicht“, erklärte Kiechle. In der Folge erhalten $20-40 \%$ der Patientinnen eine Übertherapie. Um dieses Problem zu umgehen, wurden in den letzten Jahren verstärkt neue Parameter gesucht. Genexpressionstest haben laut Kiechle das Potenzial, das diagnsotische Dilemma zu lösen. Drei Genexpressionstests sind derzeit auf dem Markt:

\section{Mammaprint: basiert auf 70 Genen, nur} auf Kryogewebe durchführbar, Analyse in Amsterdam, identifiziert Patientinnen mit niedrigem Risiko (Metastasen-Risiko in 10 Jahren $<10 \%$ ) ohne Systemtherapie; Kosten: 2.675 Euro.

2. Oncotype DX: basiert auf 23 Genen, auf fixiertem Gewebe durchführbar, Analyse in den USA, identifiziert Patientinnen mit niedrigem Risiko (FernmetastasenRisiko in 10 Jahren $<10 \%$ ) unter Tamoxifen-Therapie; Kosten: 2.600 Euro.

3. EndoPredict: der neueste Test (von 2011), basiert auf 12 Genen, auf fixiertem Gewebe durchführbar, Analyse in Deutschland und innerhalb von einem Tag, identifiziert Patientinnen mit niedrigem Risiko (Metastasen-Risiko in 10 Jahren $<10 \%$ ) unter endokriner Therapie, Kosten: 1.800 Euro.

Kiechle legte die Vorteile des EndoPredictScores dar: Er ist ausschließlich bei Hormonrezptor-positiven/HER2-negativen Patientinnen unter endokriner Therapie validiert sowie für Patientinnen mit 1-3 befallenen Lymphknoten. Er liefere prognostische Zusatzinformationen zu den anerkannten Risikofaktoren [Filipits et al. Clin Cancer Res 2011]. Ferner übertreffe die prognostische Aussagekraft des EndoPredict $_{\text {clin }}$-Score - die Kombination aus
EndoPredict und klassischen klinischen Markern (Nodalstatus, Tumorgröße) - die Aussagekraft aller konventionellen Risikofaktoren. Mit diesem Test werde die Trennschärfe zwischen den Risikogruppen noch genauer, so Kiechle, auch Patientinnen, die keine Chemotherapie brauchen, könnten damit identifiziert werden. Noch fehlen jedoch prospektive Daten, schränkte Kiechle ein. Dr. Claudia Mäck

3. Hauptthema "Neues aus der Onkologie", 8. März 2012

Mehr vom FOKO auf springermedizin.de

- Sicher verhüten in der Perimenopause - so geht's (2839018)

- Gynäkologische Versorgung von Frauen mit Behinderung (2367470)

- Frauen rechtzeitig vor Gewichtszunahme warnen (2367032)

- Diese Artikel finden Sie, indem Sie den Titel oder die in Klammern gesetzte ID-Nummer in die Suche eingeben. 